\title{
Erratum to: Validation of the Spanish version of the borderline symptom list, short form (BSL-23)
}

Joaquim Soler ${ }^{1,2^{*}}$, Daniel Vega ${ }^{3,4}$, Albert Feliu-Soler ${ }^{1,2}$, Joan Trujols ${ }^{1,2}$, Ángel Soto ${ }^{3}$, Matilde Elices ${ }^{1}$, Cristina Ortiz ${ }^{1}$, Víctor Pérez ${ }^{1,2}$, Martin Bohus ${ }^{5}$ and Juan Carlos Pascual ${ }^{1,2}$

\section{Erratum}

The original article [1] is missing some funding information from the Acknowledgements section. The authors would like to state the following instead:

This study was supported by Centro de Investigación Biomédica en Red de Salud Mental (CIBERSAM) and by a grant from Instituto de Salud Carlos III (PI10/00253), co-financed by the European Regional Development Fund (ERDF).

\footnotetext{
Author details

${ }^{1}$ Servicio de Psiquiatría, Hospital de la Santa Creu i Sant Pau, Universitat Autònoma de Barcelona, Avda Sant Antoni Ma Claret 167, Barcelona 08025, Spain. ${ }^{2}$ Centro de Investigación Biomédica en Red de Salud

Mental(CIBERSAM), Madrid, Spain. ${ }^{3}$ Servei de Psiquiatria i Salut Mental, Hospital de Igualada (Consorci Sanitari de I'Anoia), Igualada, Spain. ${ }^{4}$ Unitat de Psicologia Mèdica, Departament de Psiquiatria i Medicina Legal \& Institut de Neurociències, Universitat Autònoma de Barcelona, Barcelona, Spain. ${ }^{5}$ Department of Psychosomatics and Psychotherapy, Central Institute of Mental Health, Manheim, Germany.
}

Received: 23 November 2016 Accepted: 29 November 2016 Published online: 01 December 2016

\section{References}

1. Soler J, Vega D, Feliu-Soler A, Trujols J, Soto A, Elices M, Ortiz C, Pérez V, Bohus M, Pascual JC. Validation of the Spanish version of the borderline symptom list, short form (BSL-23). BMC Psychiatry. 2013;13:139. doi:10.1186/1471-244X-13-139.

\footnotetext{
* Correspondence: jsolerri@santpau.cat

${ }^{1}$ Servicio de Psiquiatría, Hospital de la Santa Creu i Sant Pau, Universitat Autònoma de Barcelona, Avda Sant Antoni Ma Claret 167, Barcelona 08025, Spain

${ }^{2}$ Centro de Investigación Biomédica en Red de Salud Mental(CIBERSAM), Madrid, Spain
} 